This is the accepted manuscript of:

Pizza F, Antelmi E, Vandi S, Meletti S, Erro R, Baumann CR, Bhatia KP, Dauvilliers Y, Edwards MJ, Iranzo A, Overeem S, Tinazzi M, Liguori R, Plazzi G. The distinguishing motor features of cataplexy: a study from video-recorded attacks. Sleep. 2018 May 1;41(5).

Final peer reviewed version available at: https://doi.org/10.1093/sleep/zsy026

Rights / License:

The terms and conditions for the reuse of this version of the manuscript are specified in the publishing policy. For all terms of use and more information see the publisher's website.

This item was downloaded from IRIS Università di Bologna (https://cris.unibo.it/)

When citing, please refer to the published version. 


\section{The distinguishing motor features of cataplexy: a study from video recorded attacks}

Fabio Pizza, MD, PhD ${ }^{1,2}$; Elena Antelmi, $\mathrm{MD}^{1,2}$; Stefano Vandi, $\mathrm{RPSGT}^{1,2}$; Stefano Meletti, MD, PhD ${ }^{3}$; Roberto Erro, MD ${ }^{4,5}$; Christian R. Baumann, $\mathrm{MD}^{6}$; Kailash P. Bhatia, $\mathrm{MD}^{4}$; Yves Dauvilliers, MD, PhD ; Mark J Edwards, FRCP, PhD ${ }^{4,8}$; Alex Iranzo, $\mathrm{MD}^{9}$; Sebastiaan Overeem, $\mathrm{MD}^{10,11}$; Michele Tinazzi, MD, PhD ${ }^{12}$; Rocco Liguori, MD ${ }^{1,2}$; Giuseppe Plazzi, MD ${ }^{1,2}$.

1 - Department of Biomedical and Neuromotor Sciences, University of Bologna, Bologna, Italy;

2 - IRCCS Institute of the Neurological Sciences, Ospedale Bellaria, AUSL di Bologna, Bologna, Italy; 3 Department of Biomedical, Metabolic, and Neural Sciences, University of Modena and Reggio Emilia, 41100 Modena, Italy; N.O.C.S.A.E. Hospital, AUSL 41100 Modena, Italy; 4 - Sobell Department of Neuroscience and Movement Disorders, Institute of Neurology, University College London, London, United Kingdom; 5 Center for Neurodegenerative Diseases (CEMAND), Department of Medicine, Neuroscience section, University of Salerno, Italy; 6 - Department of Neurology, University Hospital Zurich, Frauenklinikstrasse 26, CH-8091 Zurich, Switzerland; 7 - Reference National Center for Narcolepsy, Sleep Unit, Department of Neurology, Gui-de-Chauliac Hospital, University of Montpellier 1, Montpellier, INSERM U1061, France; 8 - St George's University of London, Cranmer Terrace, London SW17 ORE, United Kingdom; 9 - Hospital Clinic de Barcelona, Neurology Service, Multidisciplinary Sleep Unit, IDIBAPS, CIBERNED, Barcelona, Spain; 10 - Sleep Medicine Center Kempenhaeghe, Heeze, the Netherlands; 11 - Eindhoven University of Technology, Eindhoven, the Netherlands; 12 - Department of Neuroscience, Biomedicine and Movement Science, University of Verona, Verona, Italy. 
Institution where the work was performed: Department of Biomedical and Neuromotor Sciences, University of Bologna, Bologna, Italy.

\section{Corresponding Author:}

Giuseppe Plazzi

Department of Biomedical and Neuromotor Sciences, Ospedale Bellaria, Padiglione G

Via Altura 3, 40139 Bologna, Italy. Phone: +39 $0514966926 \quad$ Fax: +39 0514966176

Mail: giuseppe.plazzi@unibo.it

\section{Abstract}

Objectives: To describe the motor pattern of cataplexy and to determine its phenomenological differences from pseudocataplexy in the differential diagnosis of episodic falls.

Methods: We selected 30 video recorded cataplexy and 21 pseudocataplexy attacks in 17 and 10 patients evaluated for suspected narcolepsy and with final diagnosis of narcolepsy type 1 and conversion disorder respectively, together with self-reported attacks features, and asked expert neurologists to blindly evaluate the motor features of the attacks. Video documented and self-reported attacks features of cataplexy and pseudocataplexy were contrasted.

Results: Video-recorded cataplexy can be positively differentiated from pseudocataplexy by the occurrence of facial hypotonia (ptosis, mouth opening, tongue protrusion) intermingled by jerks and grimaces abruptly interrupting laughter behavior (i.e. smile, facial expression) and postural control (head drops, trunk fall) under clear emotional trigger. Facial involvement is present in both partial and generalized cataplexy. Conversely, generalized pseudocataplexy is associated with persistence of deep tendon reflexes during the attack. Self-reported features confirmed the important role of positive emotions (laughter, telling a joke) in 
Keywords: cataplexy, pseudocataplexy, cataplectic facies, functional neurological symptom disorder, narcolepsy

Statement of significance: Cataplexy assessment is exclusively based on clinical interview, possibly challenging the correct diagnosis of narcolepsy. An abrupt facial involvement of hypotonia interrupting laughter behavior marked cataplexy versus psudocataplexy in video documented attacks of patients with narcolepsy type 1 and functional neurological symptoms. Attacks of episodic fall/collapse should be documented because video recorded attacks provide reliable information useful for phenotyping ambiguous cases.

\section{Introduction}

Narcolepsy type 1 (NT1) is a central hypersomnia linked to the loss of hypothalamic hypocretinergic neurons. ${ }^{1}$ Cataplexy is pathognomonic for NT1 and is characterized, as per consensus criteria, by episodes of brief, symmetrical, loss of muscle tone with retained consciousness precipitated by strong emotions. Cataplexy "needs to be established based on the clinical interview alone because patients are rarely examined during an attack" ${ }^{1}$ However, a larger phenotypic spectrum exists encompassing between and within patients variability of the role of triggers, ${ }^{2,3}$ hypotonia distribution (partial and generalized), frequency and duration of the attacks. ${ }^{3}$ 
This clinical variability challenges the differential diagnosis. Up to $46 \%$ of non-narcoleptic subjects report "episodes of muscle weakness with various emotions or athletic activities", ${ }^{2}$ a phenomenon resembling "weakness with laughter" in the general population. ${ }^{4}$ A difficult differential diagnosis is conversion disorder (functional neurological symptom disorder, FNSD) with mixed symptoms, ${ }^{5,6}$ to be clinically classified as pseudonarcolepsy ${ }^{1}$ and pseudocataplexy (i.e. psychogenic non cataplectic attack) ${ }^{7,8}$ when the complaints are not objectively confirmed.

Few studies documented cataplexy, showing three sequential phases: "initial" with eye closure, knees buckling, and twitches; "falling" with smile interruption, jaw sagging, rhythmic postural lapses and fall; and "atonic" laying on the ground. ${ }^{9}$ Similar findings were confirmed in a larger NT1 population, ${ }^{10}$ while in childhood NT1 at disease onset a particular facial involvement ("cataplectic facies") occurred spontaneously in the context of a complex movement disorder that vanished during disease course. ${ }^{11-13}$

Hence, we aimed comparing the phenomenology of cataplexy in adult NT1 patients to identify discriminating features from pseudocataplexy as documented by video recordings, a not expensive and widely available tool.

\section{Methods}

The clinical population included patients referred by other neurologists, general practitioners or by themselves to the Center for Narcolepsy (University of Bologna) for suspected NT1 after triage based on the presence of subjective complaints of excessive daytime sleepiness and falls during emotional stimulation.

All patients were hospitalized in drug free condition (drug withdrawal since at least three weeks) to undergo a standardized assessment that included subjective sleepiness assessment with the Epworth Sleepiness Scale (ESS) ${ }^{14}$ 48-hour polysomnography, video-polygraphic documentation of suspected 
cataplectic attacks, multiple sleep latency test (MSLT), blood drawn and lumbar puncture for HLADQB1*06:02 typing and hypocretin-1 determination. ${ }^{15}$

The diagnosis of NT1 was in accordance with current criteria, ${ }^{1}$ further requiring for inclusion in the study the following criteria: i) presence of cerebrospinal hypocretin-1 levels below $110 \mathrm{pg} / \mathrm{mL}$ and of the HLADQB1*06:02 allele ii) documented chin atonia during cataplexy, and iii) absence of coexistent or documented pseudocataplexy. The diagnosis of FNSD was based on the exclusion of a neurological disorder by means of the following criteria: i) normal polysomnographic findings ruling out narcolepsy; ii) normal cerebrospinal hypocretin-1 levels; and iii) normal muscle tone during documented attacks. Three FNSD and 5 NT1 patients not presenting attacks, and one NT1 patient showing both cataplectic and psudocataplectic attacks were excluded from the study leading to a clinical population of 27 subjects. All FNSD patients were referred to psychiatric department after our hospitalization for further diagnostic and therapeutic purposes.

The study was approved by the ethical standards committee (Comitato Etico Interaziendale Bologna-Imola, number 17009), and patients signed a written informed consent including the publication of videos and photographs.

\section{Self-reported Features of Cataplexy}

A semi-structured interview was performed to ascertain specific features of attacks: 1) episode frequency $(<1 / \mathrm{yr}, 1 / \mathrm{yr}-1 /$ month, $1 /$ month-1/week, $1 /$ week-1/day , or $>1 /$ day $\left.) ;{ }^{16} 2\right)$ triggering role of emotions (no apparent emotion, laughter, telling a joke, being angry, being startled, or unexpectedly meeting an acquaintance); 3) presence of prodromal symptoms (open question); 4) potential favouring role of other factors (open question); 5) pattern of reported muscle weakness (whole body, face/jaw, neck with head drop, upper and/or lower limbs); 6) presence of preserved consciousness through the attack (defined on the basis of recalling events occurring during the attacks); 7) presence of additional ictal motor features (i.e. 
twitches, jerks); 8) duration of the majority of the attacks ( $<10 \mathrm{~s}, 10 \mathrm{~s}-2 \mathrm{~min}, 2 \mathrm{~min}-10 \mathrm{~min}$, or $>10 \mathrm{~min})$; and 9) subjective feeling of malaise at attacks' end.

\section{Video recording procedures and attack selection}

As previously detailed, ${ }^{11-13}$ cataplexy documentation was systematically performed in laboratory under polysomnographic recording. ${ }^{15}$ Each patient was interviewed to determine humor preference, and the video-recording was performed with the subject sitting and/or standing for 10 to $40 \mathrm{~min}$ ( 5 min baseline, up to 30 min under stimulation). In case of a prolonged generalized attack, deep tendon reflexes were tested. At the end, patients were asked if the attacks they experienced were typical of their habitual attacks, confirmed attacks occurrence on video recordings, and rated their intensity (mild, moderate, severe). Video segments were selected for further analysis if there was chin EMG atonia or maintained muscle tone in cataplexy and pseudocataplexy, respectively.

We selected for each patient the most representative attacks making 51 video clips anonymously coded with a number. The selection included 21 pseudocataplexy (10 FNSD patients) and 30 cataplexy (17 NT1 patients).

For each video, we identified the following features: 1 ) time of attacks onset and offset; 2) episode duration; 3) topographic distribution of hypotonia; and 4) occurrence of active movements. The attacks were subclassified as a) partial, when hypotonic phenomena across the whole video clip occurred mainly in the face and neck (encompassing ptosis, tongue protrusion, head drop), upper or lower limb drop; b) generalized, when hypotonia appeared generalized leading to globally impaired postural control and/or falls. 


\section{Blinded Video Evaluation}

We designed a scoring sheet based on previous studies, ${ }^{2,3,7,9,10,12,13}$ and we asked to a panel of eight European neurologists expert in the fields of narcolepsy and movement disorders to characterize each attack.

The scoring sheet consisted of two main parts (supplementary material). In the first part, each scorer had to assess the presence or absence of the following motor phenomena: 1) ptosis; 2) hypotonic mouth opening; 3) hypotonic tongue protrusion; 4) abrupt smile interruption; 5) abrupt interruption of a facial expression; 6) facial jerks; 7) facial contractions; 8) head drop; 9) trunk fall; 10) global fall; 11) exploratory look before falling; 12) active movements during hypotonia; 13) persistence of deep tendon reflexes during hypotonia (if tested); 14) clear emotional trigger; 15) abrupt recovery; 16) slow recovery; and 17) unilateral involvement. In the second part, the scorer had to judge the attack (cataplexy versus pseudocataplexy). To perform the scorings, each expert was asked to sequentially evaluate the video clips considering the possibility that each patient could have both attacks caused by cataplexy and pseudocataplexy. ${ }^{7}$ Each scorer was blind to patients features, to final diagnosis, to the number of subjects of each group, and did not have any audio translation from Italian.

\section{Statistical analysis}

For each motor phenomenon and for final episode evaluation inter-rater agreement was tested by means of Intraclass Correlation Coefficient (ICC), where a coefficient $<0.4$, between 0.40 and 0.59 , between 0.60 and 0.74 , and between 0.75 and 1 indicates a poor, fair, good, and excellent agreement respectively. For each motor phenomenon a score ranging from 0 to 8 was calculated by summing the positive evaluations provided by all scorers. Data were explored by means of descriptive statistics (mean \pm standard deviation) and frequency (\%) in each subgroup of patients and attack type. 
Statistical comparisons were performed by means of Mann-Whitney $U$ and Chi-Square tests, and the potential diagnostic value of self-reported and video documented attacks features was further explored by means of Receiver Operating Characteristics (ROC) curve analysis.

A p-value $<0.05$ was considered statistically significant.

\section{Results}

\section{Patients}

Clinical and polysomnographic features are reported in table 1 . The two groups did not differ with respect to age at symptom onset, age at evaluation, sex distribution, BMI, ESS, or to occurrence of symptoms suggesting sleep paralysis. NT1 patients (age range 8-63, mean disease duration of 8.9 years) more frequently complained of hypnagogic hallucinations, and FNSD patients more frequently reported previous treatment with antidepressants.

As expected, NT1 patients carried the HLA-DQB1*06:02 allele more frequently than patients with FNSD, were all hypocretin-1 deficient, and had shorter mean sleep latency and more frequent sleep onset REM periods at the MSLT.

\section{Self-reported features of muscle weakness episodes}

Individual self reported attacks features are reported in table 2.

Generalized attacks and additional motor features occurred in both groups, but attacks of partial weakness were more frequently reported by NT1 patients in all body areas.

Reported attack duration did not significantly differ between groups. NT1 patients reported more frequent attacks than FNSD patients. FNSD patients more frequently reported impaired level of consciousness during 
the attacks. Attacks more frequently occurred without apparent triggers in the FNSD group, positive emotions more commonly triggered the attacks in NT1 patients, while the other triggering situations did not differ between the groups.

FNSD patients more frequently reported a warning preceding the attack and persistence of malaise (mostly tiredness and asthenia) at the end of an attack.

\section{The body distribution and temporal motor pattern of individual spells}

Supplemental table 1 summarizes for each attack the temporal pattern of involvement of different body segments.

Briefly, in cataplexy attacks the video showed facial hypotonia (either as ptosis, mouth opening, facial expression/smile interruption, global facial hypotonia) in 13 out of 14 partial attacks, and in all 16 episodes of generalized cataplexy. Among the latter, in five attacks facial involvement preceded head drops and limbs/trunk involvement by several seconds, with subsequent postural dyscontrol and/or fall to the ground. In the other 11 generalized cataplectic attacks, hypotonia spread from the face towards the trunk and limbs over a very brief timeline, requiring slow-motion to capture a rostro-caudal cascade (Figure 1, Video 1). A period of immobility after the fall was observed in 6 attacks. Twenty-five out of 30 cataplexy attacks occurred during laughter.

In pseudocataplexy attacks, the motor pattern showed a large inter-individual variability. In 10 out of 21 attacks (four partial, six generalized) an eye closure resembling ptosis could be observed, but represented an active muscular contraction synkinetic to perioral muscles during smiling (Figure $2 \mathrm{~A}$ ). A common feature was trunk or head swaying in 10 out of 21 attacks, a finding paralleled by concomitant active movements or maintenance of active postures in partial or generalized attacks. When generalized pseudocataplexy attacks were characterized by prolonged immobility (9 videos), deep tendon reflexes were present and patients 
further showed large limb jerks and unusual facial grimacing (suggesting pain) (Video 2). Ten out of 21 psudocataplexy attacks occurred during laughter.

\section{Video documented features: episode number and duration}

Out of the 30 cataplexy attacks, 14 videos showed partial attacks (mean duration of $20.64 \pm 22.71 \mathrm{~s}$ ), and 16 videos presented generalized attacks (mean duration of $78.75 \pm 145.93 \mathrm{~s}$ ). NT1 patients rated their partial and generalized attacks respectively as mild ( $92.9 \%$ vs $12.5 \%)$, moderate $(7.1 \%$ vs $43.8 \%)$, and severe ( $0 \%$ vs 43.8\%). Attacks duration was significantly shorter $(p=0.021)$, and subjectively classified as milder $(p<0.0005)$ in partial versus generalized episodes.

Out of 21 pseudocataplexy attacks, 10 videos showed partial attacks (mean duration of $5.37 \pm 7.98 \mathrm{~s}$ ), and 11 videos presented generalized attacks (mean duration of $67.36 \pm 65.41 \mathrm{~s}$ ). FNSD patients self-rated their partial and generalized attacks respectively as mild ( $70 \%$ vs $9.1 \%)$, moderate ( $10 \%$ vs $36.4 \%)$, and severe ( $20 \%$ vs $54.5 \%)$. Partial attacks were significantly shorter in duration $(p=0.001)$, and subjectively classified as milder $(p=0.016)$ than generalized ones.

Partial attacks were significantly prolonged in NT1 vs FNSD patients $(p=0.009)$, whereas generalized attacks showed comparable duration in the two groups. The two groups did not differ for their subjective intensity evaluation of either attack type.

\section{Motor features of pseudocataplexy and cataplexy}

Table 3 shows the scores of elementary motor phenomena evidenced by the scorers in pseudocataplectic and cataplectic attacks, and the results of ICC for each item as well as between groups comparisons.

Cataplexy attacks were characterized by significantly higher occurrence of ptosis, hypotonic mouth opening, hypotonic tongue protrusion, abrupt interruption of a smile and of a facial expression (Figure 2B), facial jerks and grimaces, head drop, trunk fall, and presence of a clear emotional trigger as well as lower 
persistence of deep tendon reflexes during hypotonia compared to pseudocataplexy. Exploratory looking around before falling showed a non-significant trend towards higher occurrence in pseudocataplexy attacks. All other motor phenomena showed comparable scores in the two groups.

ICC analysis showed an excellent agreement between scorers judgement (Crombach Alpha of 0.883). The scorer judgement differed significantly in the two patients groups. Indeed, the scorers labelled as pseudocataplectic $81.2 \pm 19.7 \%$ and $21.2 \pm 26.3 \%$ of the attacks in the FNSD and NT1 groups, respectively $(p<0.0001)$. Similarly, the scorers labelled as cataplectic $18.8 \pm 19.8 \%$ and $78.7 \pm 26.3 \%$ of the attacks in the FNSD and NT1 groups $(\mathrm{p}<0.0001)$.

\section{Diagnostic utility of self-reported and video-documented features: ROC Curve Analysis}

We finally tested with ROC curves analysis the diagnostic accuracy of self-reported and video-documented features to identify cataplexy at the individual and attack level respectively. Table 4 summarizes significant results.

Considering self-reported features, the trigger laughter, the trigger telling a joke, as well as the involvement of the face, of the neck/head, of the lower limbs, and of the upper limbs showed a potential to identify cataplexy versus pseudocataplexy.

Considering video-documented features, the evidence of ptosis, head drop, trunk fall, clear emotional trigger, facial jerks, mouth opening, facial grimaces, abrupt smile interruption, and abrupt facial expression interruption showed a potential to identify cataplexy versus pseudocataplexy. Moreover, the combination of the hypotonic facial phenomena (ptosis, mouth opening and tongue protrusion), of abrupt facial changes (smile and facial expression interruption), of facial movements (jerks and grimaces / contractions), but not of postural dyscontrol (head drop and trunk fall), showed better performances compared to each single item in order to discriminate between cataplexy and pseudocataplexy. Finally, the combination of all the 
above with clear emotional trigger disclosed the best performances, comparably to those of combined abrupt facial changes.

\section{Discussion}

In this study, we found that the occurrence of hypotonic phenomena in the face and neck associated with facial jerks and grimaces were key motor features of cataplexy also in adult patients. The association of hypotonic phenomena (ptosis, mouth opening, tongue protrusion, head drop) with abrupt cessation of smile/facial expression, superimposed active motor features (facial jerks and grimacing), and the presence of clear emotional triggers best identified cataplexy compared to each single item. Conversely, selfreported features largely overlapped between groups and may be biased.

The pitfalls of "history taking" versus video documentation

Although current consensus criteria suggest that cataplexy can be determined by clinical interview alone, ${ }^{1}$ we showed a large overlap between attacks descriptions and sleepiness features in FNSD and NT1.

Definitely distinguishing attacks based on history taking (subjective symptoms collection) alone is therefore difficult, and can be misleading in patients presenting both attack types. ${ }^{6}$

Expert neurologists correctly categorized about $80 \%$ of the attacks suggesting the utility of video recordings in reaching a proper diagnosis. Future inter-observer reliability approaches will clarify the diagnostic accuracy of this approach combining different pieces of evidence (from history to videos) into potential scales to be compared with gold standard diagnostic tools (neurophysiology, cerebrospinal hypocretin-1 assay, and HLA-DQB1*0602 determination) that should be regarded as definite disease markers. ${ }^{17}$ Current knowledge mainly stems from studies based on questionnaires, ${ }^{2,3}$ and the classical description of cataplexy is continuously being enriched. ${ }^{12,13}$ NT1 is a rare disease, ${ }^{18}$ and clues obtained from the direct phenomena observation will help to guide history taking and to evaluate self-documented spells, as long as in clinical 
practice patients increasingly come to the outpatient clinic with video documentations, and as not all laboratories have appropriate diagnostic facilities. Expanding cataplexy knowledge will further curtail the long diagnostic delay, ${ }^{19,20}$ and will reduce the gap between prevalence estimates and diagnostic rates. Moreover, the systematic objective assessment of cataplexy can unravel "a minima" phenomena not subjectively perceived and reported by NT1 patients.

The 'cataplectic face': the breakdown of emotional facial expression

Craniocervical musculature involvement is very typical for both partial and generalized cataplexy in NT1 patients of all ages, and is usually the first motor sign as the attack develops. An emotional smile resulting from the contraction of the zygomatic muscles and of the tonic contraction of the pars lateralis of the orbicularis oculi, the so called "Duchenne smile", ${ }^{21}$ is rapidly reversed by hypotonic ptosis/mouth opening and intermingled by active facial contractions (jerks, grimaces). These facial signs are early and reliable markers of cataplexy in NT1 patients of all ages, thus extending our previous observation on the "cataplectic facies" in children. ${ }^{11-13}$

The facial musculature is the principal system to enact emotions and the main target of cataplexy. Brain correlates of humor-induced cataplexy in young NTI patients investigated by means of video-assisted fMRI localized increased neural activity in the amygdala, insular cortex, and in the ventromedial prefrontal/anterior cingulate cortex. ${ }^{22}$ These regions are part of the "amygdala-motor pathway" for the control of facial expressions. ${ }^{23}$ In healthy humans, hypocretinergic neurons are active under conditions of high arousal and positive emotions, ${ }^{24,25}$ counterbalancing any inhibition of brainstem regions at the ventrolateral periacqueductal gray/lateral pontine tegmentum. ${ }^{26}$ With the loss of hypocretin signaling in NT1, the inhibitory projections from the amygdala would fire unopposed resulting in cataplexy.

The facial involvement during cataplexy in NT1 patients of all ages also points to the role of hypocretin neurons in facilitating motoneuron activity along the neuraxis, as a high hypocretinergic innervation has been demonstrated in the brainstem nuclei coordinating eye-lid opening (levator palpebrae), ${ }^{27}$ mastication 
(trigeminal motor nucleus), ${ }^{28}$ and hypoglossal nucleus. ${ }^{29}$ Moreover, hypocretin transmission increases prominently with positive emotions and social interactions suggesting a role in proactive behaviors. ${ }^{30}$

\section{Psychiatric morbidity: mind and brain}

In the nineteenth century scientists debated on the nature of narcolepsy and contrasted former interpretation of a disease sui generis or a syndrome with different possible etiologies, including psychiatric/psychological ones. ${ }^{31}$ Our data strengthen the importance to base cataplexy and pseudocataplexy diagnoses on positive clinical signs, given the frequent occurrence of comorbid anxiety, ${ }^{32}$ mood, ${ }^{33,34}$ and psychotic disorders in NT1, ${ }^{35}$ as well as of pseudocataplexy in both $\mathrm{NT} 1,{ }^{6}$ and psychiatric patients. $^{8}$

\section{Limitations}

We acknowledge the following study limitations calling for further research in the field. First, we selected attacks from patients who showed only cataplexy (in NT1) or pseudocataplexy (in FNSD), thus preventing any potential comparison between cataplexy and pseudocataplexy coexisting in NT1. Second, a formal psychiatric assessment has not been included in the current study design and was performed after our hospitalization excluding a neurological disorder in FNSD patients to further assess psychiatric morbidity and start adequate disease management (data not available). Future studies should integrate neurological and psychiatric evaluations to further investigate the relation of different disease aspects and attacks semiology. 


\section{Conclusions}

To summarize, we showed that the direct observation of video documented cataplexy provides a set of positive signs useful to steer proper diagnosis, in particular hypotonia occurring in the facial region (i.e. ptosis, mouth opening, tongue protrusion) abruptly interrupting laughter behavior (i.e. abrupt interruption of a smile or of facial expression) intermingled by active movements (i.e. facial jerks and contractions/grimaces) before the occurrence of postural dyscontrol (head drop, trunk fall, or body fall) as gross motor features of cataplexy. Conversely, other red flags (persistence of deep tendon reflexes during generalized attacks) steer toward the clinical suspicion of pseudocataplexy. Clinicians should therefore consider the systematic use of video recordings to obtain objective information useful for correct attacks definition given the potential unreliability and overlap of subjective symptoms collection.

Abbreviation list: Narcolepsy type 1, NT1; functional neurological symptom disorder, FNSD; Epworth Sleepiness Scale, ESS; Multiple Sleep Latency Test, MSLT; Intraclass Correlation Coefficient, ICC; Receiver Operating Characteristics, ROC; Body Mass Index, BMI; Human Laucocyte Antigen, HLA.

\section{Acknowledgements}

We are indebted to all the participants of the study, most notably the Italian association of narcoleptic patients (Associazione Italiana Narcolettici e Ipersonni, AIN onlus; http://www.narcolessia.it/). Without their contributions, this study would not have been possible.

\section{Disclosure Statement}

Fabio Pizza, Elena Antelmi, Stefano Vandi, Stefano Meletti, Alex Iranzo, Michele Tinazzi, and Rocco Liguori report no disclosures. Roberto Erro received consultancies from Zambon and honoraria from TEVA. Christian R. Baumann received a grant from the Swiss National Research Foundation, is supported by the 
Clinical Research Priority Program Sleep and Health of the University of Zurich, received a research grant from the HSM Program of the Canton of Zurich, and received unrestricted research grants from UCB Pharma and from AbbVie. None of these grants are related to the present work. Kailash P. Bhatia receives royalties from Oxford University Press and a stipend for MDCP editorship, holds grants from NIHR RfPB, MRC Welcome Strategic grant (WT089698), Horizon 2020 EC grant Propag-Aging, and has received honoraria/financial support to speak/attend meetings or serve on advisory boards from Ipsen, Merz, Allergan, Sun Pharma, and Teva Lundbeck pharmaceutical companies. Yves Dauvilliers has received funds for speaking, board engagements, and travel to conferences with UCB Pharma, Jazz, Theranexus, Flamel, and Bioprojet. Mark J Edwards receives grant support from the Medical Research Council and the National Institutes of Health Research UK, and has received grants and honoraria for education activities from Merz Pharma, UCB Pharma and Medtronic. Sebastiaan Overeem is supported by a VIDI research grant from the Netherlands Organization for Scientific Research (grant no. 016.116.371), received an unrestricted research grant from UCB Pharma not related to the present work, and received conference travel support from UCB Pharma, Novartis and Boehringer Ingelheim. Giuseppe Plazzi has received funds for board engagements from UCB Pharma, Jazz, and Bioprojet.

Financial Disclosures: None of the authors have financial disclosures to the research covered in this article Funding: none. 


\section{Reference List}

1. American Academy of Sleep Medicine. International Classification of Sleep Disorders. 3rd ed. Darien, IL: American Academy of Sleep Medicine. 2014.

2. Anic-Labat S, Guilleminault C, Kraemer HC, Meehan J, Arrigoni J, Mignot E. Validation of a cataplexy questionnaire in 983 sleep-disorders patients. Sleep 1999;22:77-87.

3. Overeem S, van Nues SJ, van der Zande WL, Donjacour CE, van Mierlo P, Lammers GJ. The clinical features of cataplexy: a questionnaire study in narcolepsy patients with and without hypocretin-1 deficiency. Sleep Med 2011;1:12-8.

4. Overeem S, Lammers GJ, van Dijk JG. Weak with laughter. Lancet 1999;354:838.

5. Edwards MJ, Adams RA, Brown H, Pareés I, Friston KJ. A Bayesian account of 'hysteria'. Brain 2012;135:3495-512.

6. American Psychiatric Association. Diagnostic and Statistical Manual of Mental Disorders, Fifth Edition. Washington, DC: American Psychiatric Association; 2013.

7. Plazzi G, Khatami R, Serra L, Pizza F, Bassetti CL. Pseudocataplexy in narcolepsy with cataplexy. Sleep Med 2010;11:591-4.

8. Pizza F, Vandi S, Poli F, et al. Narcolepsy with cataplexy mimicry: the strange case of two sisters. J Clin Sleep Med 2013;9:611-2.

9. Rubboli G, d'Orsi G, Zaniboni A, et al. A video-polygraphic analysis of the cataplectic attack. Clin Neurophysiol 2000;111 Suppl 2:S120-8.

10. Vetrugno R, D'Angelo R, Moghadam KK, et al. Behavioural and neurophysiological correlates of human cataplexy: a video-polygraphic study. Clin Neurophysiol 2010;121:153-62. 
11. Serra L, Montagna P, Mignot E, Lugaresi E, Plazzi G. Cataplexy features in childhood narcolepsy. Mov Disord 2008;23:858-65.

12. Plazzi G, Pizza F, Palaia V, et al. Complex movement disorders at disease onset in childhood narcolepsy with cataplexy. Brain 2011;134:3477-89.

13. Pizza F, Franceschini C, Peltola H, et al. Clinical and polysomnographic course of childhood narcolepsy with cataplexy. Brain 2013;136:3787-95.

14. Johns MW. A new method for measuring daytime sleepiness: the Epworth sleepiness scale. Sleep $1991 ; 14: 540-5$.

15. Pizza F, Moghadam KK, Vandi S, et al. Daytime continuous polysomnography predicts MSLT results in hypersomnias of central origin. J Sleep Res 2013;22:32-40.

16. Dauvilliers $\mathrm{Y}$, Montplaisir J, Molinari N, et al. Age at onset of narcolepsy in two large populations of patients in France and Quebec. Neurology 2001;57:2029-33.

17. Vignatelli L, Bisulli F, Provini F, et al. Interobserver reliability of video recording in the diagnosis of nocturnal frontal lobe seizures. Epilepsia 2007;48:1506-11.

18. Longstreth WT Jr, Koepsell TD, Ton TG, Hendrickson AF, van Belle G. The epidemiology of narcolepsy. Sleep 2007;30:13-26.

19. Thorpy MJ, Krieger AC. Delayed diagnosis of narcolepsy: characterization and impact. Sleep Med 2014;15:502-7.

20. Luca G, Haba-Rubio J, Dauvilliers Y, et al. Clinical, polysomnographic and genome-wide association analyses of narcolepsy with cataplexy: a European Narcolepsy Network study. J Sleep Res 2013;22:482-95.

21. Ekman P, Davidson RJ, Friesen WV. The Duchenne smile: emotional expression and brain physiology. II. J Pers Soc Psychol 1990;58:342-53. 
22. Meletti S, Vaudano AE, Pizza F, et al. The Brain Correlates of Laugh and Cataplexy in Childhood Narcolepsy. J Neurosci 2015;35:11583-94.

23. Gothard KM. The amygdalo-motor pathways and the control of facial expressions. Front Neurosci 2014;8:43.

24. Mileykovskiy BY, Kiyashchenko LI, Siegel JM. Behavioral correlates of activity in identified hypocretin/orexin neurons. Neuron 2005;46:787-98.

25. Blouin AM, Fried I, Wilson CL, et al. Human hypocretin and melanin-concentrating hormone levels are linked to emotion and social interaction. Nat Commun 2013;4:1547.

26. Dauvilliers Y, Siegel JM, Lopez R, Torontali ZA, Peever JH. Cataplexy--clinical aspects, pathophysiology and management strategy. Nat Rev Neurol 2014;10:386-95.

27. Schreyer S, Büttner-Ennever JA, Tang X, Mustari MJ, Horn AK. Orexin-A inputs onto visuomotor cell groups in the monkey brainstem. Neuroscience 2009; 164: 629-40.

28. Mascaro MB, Prosdócimi FC, Bittencourt JC, Elias CF. Forebrain projections to brainstem nuclei involved in the control of mandibular movements in rats. Eur J Oral Sci 2009; 117: 676-84.

29. Fung SJ, Yamuy J, Sampogna S, Morales FR, Chase MH. Hypocretin (orexin) input to trigeminal and hypoglossal motoneurons in the cat: a double-labeling immunohistochemical study. Brain Res 2001;903:257-62.

30. Facchinetti LD, Imbiriba LA, Azevedo TM, Vargas CD, Volchan E. Postural modulation induced by pictures depicting prosocial or dangerous contexts. Neurosci Lett 2006;410:52-6.

31. Fortuyn HA, Mulders PC, Renier WO, Buitelaar JK, Overeem S. Narcolepsy and psychiatry: an evolving association of increasing interest. Sleep Med 2011;12:714-9.

32. Fortuyn HA, Lappenschaar MA, Furer JW, et al. Anxiety and mood disorders in narcolepsy: a casecontrol study. Gen Hosp Psychiatry. 2010;32: 
49-56.

33. Dauvilliers Y, Paquereau J, Bastuji H, Drouot X, Weil JS, Viot-Blanc V. Psychological health in central hypersomnias: the French Harmony study. J Neurol Neurosurg Psychiatry 2009;80:636-41.

34. Ohayon MM. Narcolepsy is complicated by high medical and psychiatric comorbidities: a comparison with the general population. Sleep Med 2013;14:488-92.

35. Canellas F, Lin L, Julià MR, et al. Dual cases of type 1 narcolepsy with schizophrenia and other psychotic disorders. J Clin Sleep Med 2014;10:1011-8. 
Figure and Video Captions

Figure 1: frame by frame sequence of body involvement during a cataplectic attack Legend: frame by frame sequence (from left to right) of sequential body involvement during a generalized cataplectic attack showing a rostro-caudal progression from smile interruption, facial hypotonia, head drop, trunk drop and global fall.

Figure 2: active (panel A) and hypotonic (panel B) eye closure sequence during laughter Legend: In the upper panel (A) is showed an example of "Duchenne smile" with active eye closure synkinetic with smile. In the lower panel (B) is showed the frame by frame sequence (from left to right) of cataplexy with ptosis and facial hypotonia briefly interrupting laughter behavior.

Video 1: example of a generalized cataplexy attack in a patient with narcolepsy type 1

Video 2: example of a generalized pseudocataplexy attack in a functional neurological disorder patient 
Tables

Table 1: Clinical Features

\begin{tabular}{|c|c|c|c|c|c|}
\hline \multirow[t]{2}{*}{ Clinical Features } & \multicolumn{2}{|c|}{ FND $(n=10)$} & \multicolumn{2}{|c|}{ NT1 (n=17) } & \multirow[b]{2}{*}{ P-Value } \\
\hline & Mean & SD & Mean & SD & \\
\hline Age (years) & 31.70 & 15.19 & 33.00 & 18.99 & 0.763 \\
\hline Male Sex (\%) & 20.0 & & 47.0 & & 0.160 \\
\hline $\operatorname{BMI}\left(\mathrm{kg} / \mathrm{m}^{2}\right)$ & 27.18 & 5.73 & 29.34 & 6.99 & 0.616 \\
\hline Age at symptoms onset (years) & 25.30 & 15.97 & 24.06 & 13.75 & 0.920 \\
\hline Daytime Sleepiness Onset (years) & 17.67 & 9.18 & 21.18 & 11.74 & 0.805 \\
\hline Hypnagogic Hallucinations (\%) & 20.0 & & 82.4 & & 0.001 \\
\hline Sleep Paralyses (\%) & 40.0 & & 70.6 & & 0.118 \\
\hline Antidepressant Use (\%) & 40.0 & & 5.9 & & 0.028 \\
\hline HLA-DQB1*06:02 (\%) & 29.0 & & 100.0 & & 0.000 \\
\hline CSF-hcrt-1 (pg/mL) & 371.67 & 65.22 & 19.36 & 25.90 & 0.000 \\
\hline \multicolumn{6}{|l|}{ Sleep Features } \\
\hline ESS (n) & 17.50 & 17.18 & 17.20 & 3.49 & 0.348 \\
\hline Sleep Latency (min) & 12.61 & 11.86 & 4.00 & 2.15 & 0.128 \\
\hline REM Sleep Latency (min) & 66.06 & 29.62 & 43.00 & 42.52 & 0.138 \\
\hline Total Sleep Time (min) & 426.67 & 52.18 & 421.25 & 132.90 & 0.923 \\
\hline Sleep Efficiency (\%) & 88 & 6 & 80 & 14 & 0.141 \\
\hline MSLT: Sleep Latency (min) & 12.21 & 4.26 & 3.79 & 2.70 & 0.000 \\
\hline MSLT: SOREMPs number (n) & 0.22 & 0.44 & 3.87 & 1.41 & 0.000 \\
\hline
\end{tabular}

Legend: FND, functional neurological disorder; NT1, narcolepsy type 1; BMI, body mass index; HLA, human leucocyte antigen; CSF-hcrt-1, cerebrospinal hypocretin 1 level; ESS, Epworth sleepiness scale; REM, rapid eye movements; MSLT, multiple sleep latency test. 
Table 2: Self reported features

\begin{tabular}{|c|c|c|c|}
\hline Features & FND $(n=10)$ & NT1 (n=17) & P-Value \\
\hline \multicolumn{4}{|l|}{ Frequency } \\
\hline$<1 / y(\%)$ & 0.00 & 0.00 & 0.032 \\
\hline $1 / y-1 / m(\%)$ & 10.00 & 0.00 & \\
\hline $1 / m-1 / w(\%)$ & 40.00 & 5.90 & \\
\hline $1 / w-1 / d(\%)$ & 0.00 & 29.40 & \\
\hline$>1 / d(\%)$ & 50.00 & 64.70 & \\
\hline \multicolumn{4}{|l|}{ Triggers } \\
\hline Without apparent trigger (\%) & 70.00 & 23.50 & 0.018 \\
\hline Laughter (\%) & 40.00 & 100.00 & 0.000 \\
\hline Telling a Joke (\%) & 0.00 & 64.70 & 0.001 \\
\hline Being Angry (\%) & 70.00 & 52.90 & 0.500 \\
\hline Being Startled (\%) & 20.00 & 47.10 & 0.160 \\
\hline Unexpectedly Acquaitance Meeting (\%) & 30.00 & 52.90 & 0.247 \\
\hline Prodromic symptoms (\%) & 50.00 & 0.00 & 0.001 \\
\hline Favouring Factors (\%) & 10.00 & 5.90 & 0.693 \\
\hline \multicolumn{4}{|l|}{ Pattern } \\
\hline Weakness: Whole Body (\%) & 90.00 & 88.20 & 0.888 \\
\hline Weakness: Facial - Jaw (\%) & 10.00 & 94.10 & 0.000 \\
\hline Weakness: Neck - Head drop (\%) & 0.00 & 94.10 & 0.000 \\
\hline Weakness: Knees (\%) & 20.00 & 82.40 & 0.001 \\
\hline Weakness: Arms - Hands (\%) & 20.00 & 76.50 & 0.004 \\
\hline Additional Features (\%) & 30.00 & 41.20 & 0.561 \\
\hline Preserved Consciousness (\%) & 60.00 & 100.00 & 0.005 \\
\hline Additional features (\%) & 30.00 & 41.20 & 0.561 \\
\hline \multicolumn{4}{|l|}{ Duration } \\
\hline$<10$ s (\%) & 0.00 & 29.40 & 0.072 \\
\hline $10 s-2 m(\%)$ & 40.00 & 47.00 & \\
\hline
\end{tabular}




\begin{tabular}{|l|r|r|}
\hline $2 m-10 m(\%)$ & 20.00 & 17.60 \\
\hline$>10 m(\%)$ & 40.00 & 5.90 \\
\hline
\end{tabular}

Legend: FND, functional neurological disorder; NT1, narcolepsy type $1 ; 1 / \mathrm{y}$, one per year; $1 / \mathrm{m}$, one per month; $1 / w$, one per week; $1 / d$, one per day. 
Table 3: scores of motor features occurrence in video documented attacks

\begin{tabular}{|l|c|c|c|c|c|c|}
\hline & ICC Analysis & \multicolumn{2}{l|}{ FND (n=21) } & \multicolumn{2}{l|}{ NT1 (n=30) } & Mann-Whitney \\
\hline Motor features & Cronbach's Alpha & Mean & SD & Mean & SD & $p$-value \\
\hline 1.Ptosis & 0.741 & 3.43 & 1.80 & 4.90 & 2.68 & 0.0287 \\
\hline 2.Mouth opening & 0.887 & 0.86 & 1.20 & 3.43 & 2.79 & 0.0004 \\
\hline 3.Tongue protrusion & 0.859 & 0.00 & 0.00 & 0.80 & 1.61 & 0.0065 \\
\hline 4.Abrupt smile interruption & 0.767 & 0.48 & 0.60 & 3.33 & 2.01 & 0.0000 \\
\hline 5.Abrupt facial expression interruption & 0.626 & 0.67 & 0.97 & 3.10 & 1.54 & 0.0000 \\
\hline 6.Facial jerks & 0.670 & 0.24 & 0.89 & 1.40 & 1.52 & 0.0003 \\
\hline 7.Facial contractions / grimaces & 0.728 & 1.10 & 1.37 & 3.13 & 1.59 & 0.0000 \\
\hline 8.Head drops & 0.859 & 3.67 & 2.42 & 5.30 & 2.45 & 0.0200 \\
\hline 9.Trunk fall & 0.835 & 2.24 & 2.23 & 4.33 & 2.59 & 0.0058 \\
\hline 10.Global fall & 0.978 & 3.14 & 3.64 & 2.07 & 3.29 & 0.1080 \\
\hline 11.Exploratory look before falling & 0.762 & 1.00 & 1.70 & 0.27 & 0.52 & 0.0754 \\
\hline 12.Active movements during hypotonia & 0.276 & 1.76 & 1.37 & 1.97 & 0.93 & 0.2914 \\
\hline 13.Persistence of deep tendon reflexes & 0.773 & 2.52 & 2.93 & 0.70 & 1.74 & 0.0193 \\
\hline 14.Clear emotional trigger & 0.776 & 4.10 & 2.74 & 6.40 & 1.92 & 0.0012 \\
\hline 15.Abrupt recovery & 0.882 & 2.57 & 2.34 & 3.53 & 2.54 & 0.1606 \\
\hline 16.Slow recovery & 0.840 & 2.81 & 2.09 & 2.73 & 2.12 & 0.8081 \\
\hline 17.Unilateral involvement & 0.246 & 0.14 & 0.36 & 0.10 & 0.31 & 0.6434 \\
\hline
\end{tabular}

Legend: FND, functional neurological disorder; NT1, narcolepsy type 1. 
Table 4: Receiver Operating Curve (ROC) analysis results

\begin{tabular}{|c|c|c|}
\hline Features & AUC & P-Value \\
\hline \multicolumn{3}{|l|}{ Self reported features } \\
\hline Trigger: laughter & $0.78 \pm 0.11$ & 0.022 \\
\hline Trigger: telling a joke & $0.82 \pm 0.08$ & 0.008 \\
\hline Pattern: Facial - Jaw & $0.91 \pm 0.07$ & 0.001 \\
\hline Pattern: Neck - Head drop & $0.97 \pm 0.03$ & $<0.005$ \\
\hline Pattern: Knees & $0.80 \pm 0.10$ & 0.013 \\
\hline Pattern: Arms - Hands & $0.77 \pm 0.10$ & 0.025 \\
\hline \multicolumn{3}{|l|}{ Video documented features } \\
\hline Ptosis & $0.68 \pm 0.08$ & 0.031 \\
\hline Head drops & $0.69 \pm 0.08$ & 0.021 \\
\hline Trunk fall & $0.73 \pm 0.07$ & 0.006 \\
\hline Clear emotional trigger & $0.76 \pm 0.07$ & 0.002 \\
\hline Facial jerks & $0.77 \pm 0.07$ & 0.001 \\
\hline Mouth opening & $0.79 \pm 0.06$ & 0.001 \\
\hline Facial contractions / grimaces & $0.84 \pm 0.06$ & $<0.0001$ \\
\hline Abrupt smile interruption & $0.89 \pm 0.05$ & $<0.0001$ \\
\hline Abrupt facial expression interruption & $0.90 \pm 0.04$ & $<0.0001$ \\
\hline \multicolumn{3}{|l|}{ Combination of video documented features } \\
\hline A: Hypotonic face (ptosis+mouth opening+tongue protrusion) & $0.77 \pm 0.07$ & 0.001 \\
\hline B: Abrupt facial changes (smile interruption+facial expression interruption) & $0.92 \pm 0.04$ & $<0.0001$ \\
\hline C: Facial movements (jerks+contractions/grimaces) & $0.86 \pm 0.05$ & $<0.0001$ \\
\hline D: Postural dyscontrol (head drop+trunk fall) & $0.77 \pm 0.07$ & 0.001 \\
\hline$A+B+C+D+$ clear emotional trigger & $0.92 \pm 0.04$ & 0.039 \\
\hline
\end{tabular}

Legend: AUC, area under the curve. 
Figure 1.

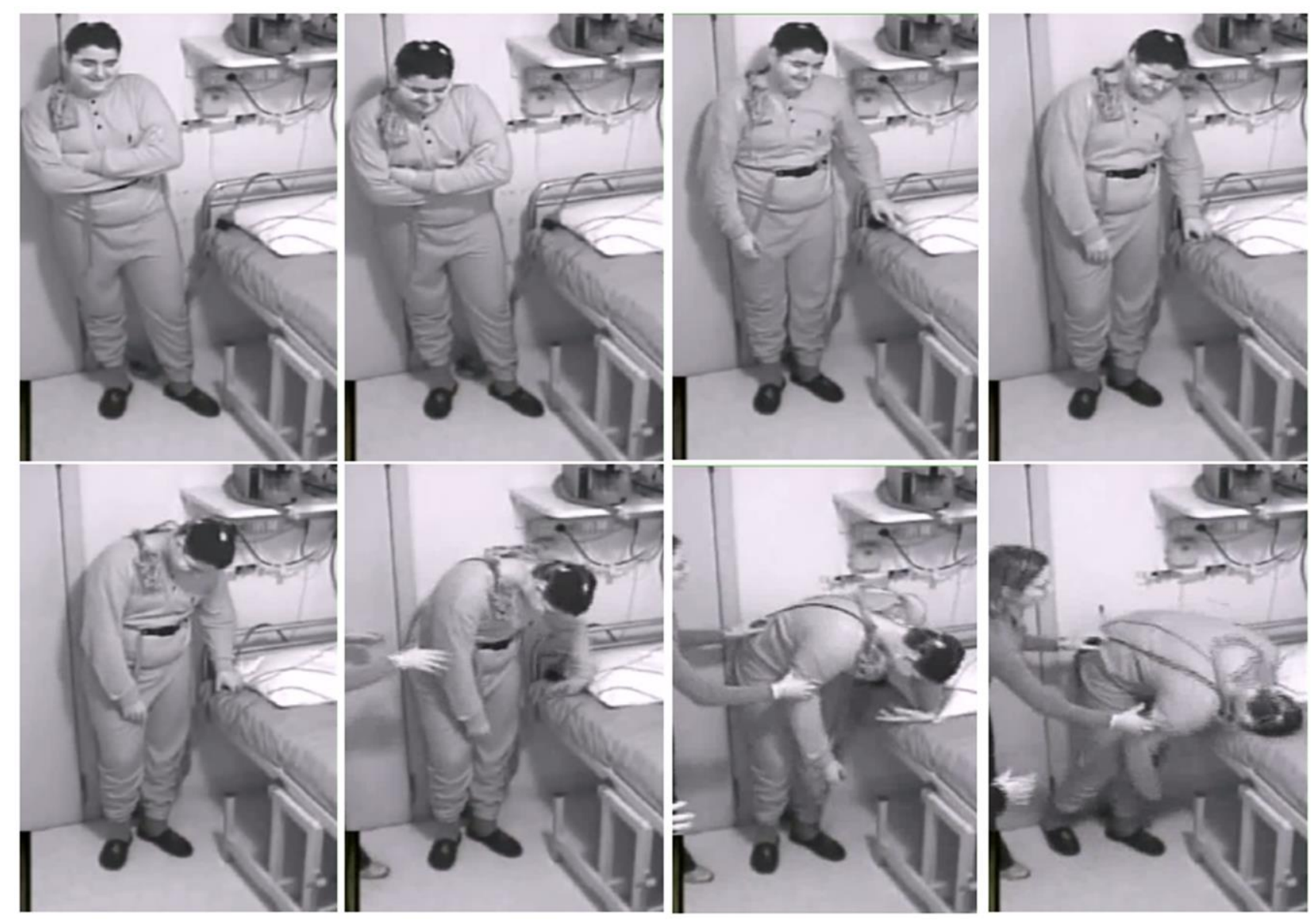


Figure 2.

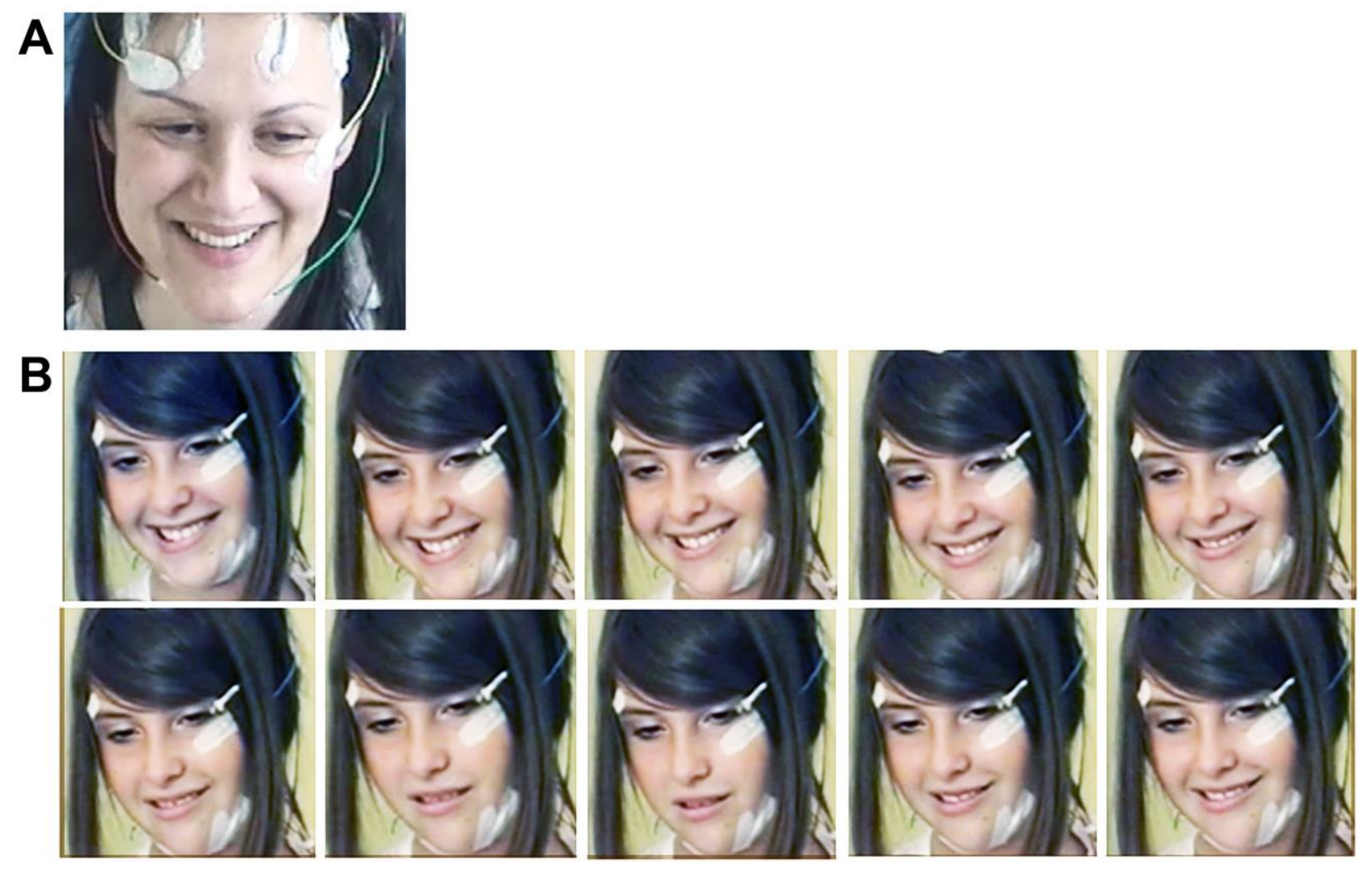

